\title{
External Disturbance Detection and Its Application on the Identification of Fault in CMG System
}

\author{
Jung-Hyung Lee, Hun-Jo Lee, Joon-Yong Lee and Hwa-Suk Oh \\ Department of Aero \& Mechanical Engineering, Korea Aerospace University, Goyang 10540, Korea
}

\begin{abstract}
CMGs (control moment gyros) as satellite actuators have intrinsic structures generating disturbance, like bearings, motors, high-speed rotating wheels, steering gimbal, etc. Disturbances induced by faults in some of these parts shall be detected immediately and identified in real time. A continuous second order sliding mode observer can be applied for the detection of disturbances. In this paper, a nonlinear sliding mode observing algorithm based on the gyro sensor is suggested for the detection of external disturbances. The algorithm is then applied to detect a fault in a CMG, here the wheel fluctuation fault. By distinguishing the direction of disturbance torque by a diagnosis algorithm, the fault CMG can be then identified and isolated from other normal CMGs. The performance of detecting algorithm is verified on the hardware satellite simulator, in which four CMGs are installed.
\end{abstract}

Key words: Satellite actuator, fault detection, identification, sliding mode observer.

\section{Introduction}

CMGs (control moment gyros) as momentum exchanging actuators, have been used as primary attitude control actuators for a variety of spacecraft. Slew or reorientation maneuvers of the spacecraft are executed by steering the gimbals of CMGs [1]. For the precision control of spacecraft, it is thus necessary that the gimbals are steered precisely while each wheel holding the nominal speed. Severe speed fluctuation either in wheel rotation or gimbal steering may induce critical disturbances [2-4]. While monitoring the spacecraft health, this type of severe faults in actuators thus need to be detected and isolated as quickly in real time in orbit as possible. Since the magnitude and frequency characteristics of such fault disturbances differ from those in normal conditions, the algorithm for the detection and identification of external disturbances can be used as a detector of actuator faults.

Since a spacecraft model is intrinsically nonlinear in large angle maneuvers, a proper nonlinear fault

Corresponding author: Hwa-Suk Oh, Ph.D., professor, research field: satellite attitude control. detection and isolation strategy should be adopted [5-7]. Second order SMOs (sliding mode observers) have been suggested in several studies [8-12]. In this paper, a second order nonlinear SMO is applied to detect CMG fault-induced disturbances. The chattering in the conventional sliding observer is avoided by using the continuous type observer law as in Ref. [11]. The magnitude and frequency of the oscillating disturbance are estimated properly. The gimbal orientation of CMGs needs to be considered when isolating faults. First, the working concept of the suggested continuous type sliding mode observer is explained theoretically. Next, the observing algorithm is applied to detect the disturbances induced by the CMG fault - the high speed wheel motor fault. A vector transpose isolation scheme in the single fault case is suggested, and the performances are proven through several simulations with hardware simulator equipped with four CMGs.

\section{Attitude Control with CMGs}

A spacecraft with four single gimbal CMGs installed in a pyramid configuration is considered as shown in Fig. 1. 


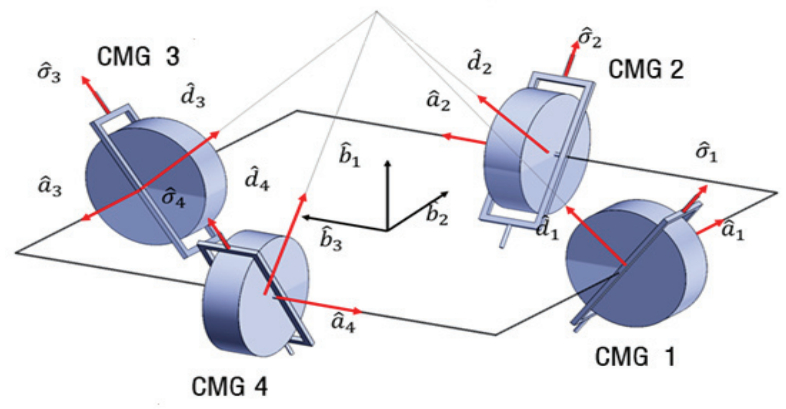

Fig. 1 CMGs installed in pyramid configuration.

The motion of the spacecraft is then governed by the following equations:

$$
\begin{gathered}
\dot{q}=\frac{1}{2} E(q) \omega \\
I \dot{\omega}+\omega^{\times} I \omega+\omega^{\times} A h=u_{C}+u_{f} \\
D \dot{\sigma}=-u_{C}
\end{gathered}
$$

where $q$ is the spacecraft attitude quaternion, $\omega$ the angular velocity, and $h=\left[\begin{array}{llll}h_{1} & h_{2} & h_{3} & h_{4}\end{array}\right]^{T}$ the wheel momentum vector. The matrix $E(q)$ is the quaternion matrix and $I$ is the spacecraft inertia matrix. Matrix $D$, composed of the output torque vectors $d_{i}$ s as $D=\left[\begin{array}{llll}d_{1} & d_{2} & d_{3} & d_{4}\end{array}\right]$, changes with the CMG gimbal angles $\sigma=\left[\begin{array}{llll}\sigma_{1} & \sigma_{2} & \sigma_{3} & \sigma_{4}\end{array}\right]^{T}$. Vector $u_{C}$ is the control torque generated by steering $\sigma$. Matrix $A$ is the gimbal configuration matrix, which is composed of the momentum wheel axis vectors $a_{i}$ s as $A=\left[\begin{array}{llll}a_{1} & a_{2} & a_{3} & a_{4}\end{array}\right]$. To go to the target attitude $q_{T}$, a conventional quaternion feedback control law Eq. (4) and a singular robust steering law Eq. (5) can be used as follows [13-15]:

$$
\begin{gathered}
u_{C}=\omega^{\times}(I \omega+A h)-K_{D} \omega \\
+K_{P} E^{T}(q) q_{T}+K_{I} \int E^{T}(q) \omega d t \\
\dot{\sigma}=-D^{T}\left(D D^{T}+\alpha\right)^{-1} u_{C}
\end{gathered}
$$

The disturbance torque vector $u_{f}$ consists of several kinds of disturbances $[11,12]$. The disturbances in this study are confined to one due to $u_{S}$, the wheel speed fluctuation disturbance. The wheel momentum $h$ is constant in normal conditions as $u_{S}=0$. However, in the wheel speed fault due to the motor control fault, the wheel momentum is disturbed by a speed fluctuation, in which case $u_{S}$ would have a nonzero value in a component corresponding to the fault wheel.

\section{Disturbance Observer Design}

When a disturbance $u_{f}$ is exerted on the spacecraft body, the spacecraft attitude is then affected immediately. A state observer can be used as a tool to monitor the disturbance. Since the attitude equations are nonlinear, a nonlinear sliding observer is considered here as $[9,11]$

$$
\widehat{I} \dot{\hat{\omega}}+\widehat{\omega}^{\times} \widehat{I} \widehat{\omega}+\widehat{\omega}^{\times} A h=u_{C}-\widehat{I} v
$$

where $\widehat{I}$ is the nominal estimate value of $I$, and $\widehat{\omega}$ of $\omega$. If the state estimate error is defined as $e=\omega-\widehat{\omega}$, then the error dynamics becomes

$$
\dot{e}=f+I^{-1} u_{f}+v
$$

where $f$ is the system error due to the error in the inertia and angular velocity as follows:

$$
f \equiv I^{-1}\left(-\omega^{\times} I \omega-\omega^{\times} A h\right)-\widehat{I}^{-1}\left(-\widehat{\omega}^{\times} \widehat{I} \widehat{\omega}-\widehat{\omega}^{\times} A h\right)
$$

The observer switching term $v$ is then designed by the second order sliding mode [8-11]. Let $S$ be a sliding surface satisfying

$$
\ddot{S}+z_{o} \dot{S}=\dot{e}+c e
$$

where $z_{o}$ and $c$ are stable gains to make $S \rightarrow 0$. Consider a Lyapunov function candidate $V$ with a positive $l$.

$$
V \equiv \frac{1}{2}\left(\dot{S}^{T} \dot{S}+S^{T} l S\right)
$$

By choosing $v$ as $v \equiv z_{o} \dot{S}-c e-l S-d \operatorname{sgn}(\dot{S})$, then $\dot{V} \leq 0$ and $S, e \rightarrow 0$ with the selection of a proper large positive gain $d$. However, since chattering exists around the $S=0$ surface in this type of algorithm, the function $\operatorname{sgn}(\dot{S})$ has been occasionally substituted with $\operatorname{sat}(\dot{S})$ [9, 10]. In this 
paper, however, we use a different continuous term (written as $d \dot{S}$ ) instead of $\operatorname{sat}(\dot{S})$, as suggested in Ref. [11]. With a sufficiently large gain in $d$, the switching law $v \equiv z_{o} \dot{S}-c e-l S-d \dot{S}$ stabilizes the observer around $e=0$ [11]. Finally, the estimate of disturbance $\widehat{u}_{f} \equiv-\widehat{I}(\widehat{f}+v)$ measures the disturbance $u_{f}$ within some error bound of the inertia.

\section{External Disturbance Detection}

\subsection{Secular Disturbance}

On orbit, the satellite occasionally experience secular disturbance torques, like gravity gradient torque, and solar pressure, etc., which induce the wheel momentum increase and finally saturation. The detection of secular disturbance in real time is thus helpful to provide proper curing actions. The observer designed above can be used as a detector of this type of disturbance. Consider an example case with a mid-class spacecraft, as MOI $I=\operatorname{diag}\{200,80,130\}$ $\left(\mathrm{kg} / \mathrm{m}^{2}\right)$. The observer and controller gains are set as $\left[\begin{array}{lll}K_{D} & K_{P} & K_{I}\end{array}\right]=\left[\begin{array}{lll}53 & 7 & 0\end{array}\right]$ and $\left[\begin{array}{llll}z_{o} & c & l & d\end{array}\right]=\left[\begin{array}{llll}0.6 & 100 & 0.6 & 1.65\end{array}\right]$.

Assume a secular disturbance $u=\left[\begin{array}{lll}0.1 & 0 & 0\end{array}\right]$ acts on $x$-axis of a satellite during time $t=(20,60) \mathrm{sec}$ as in Fig. 2c.

The angular velocity estimate $\widehat{\omega}$ in Fig. $2 \mathrm{~b}$ obtained from the observer follows well the real angular velocity $\omega$ in Fig. 2a. The disturbance by using the suggested switching term is well detected immediately at time $t=20 \mathrm{sec}$ and the magnitude is also identified exactly in Fig. 2d. The Lyapunov function $\dot{V} \rightarrow 0$ in Fig. $2 \mathrm{f}$ and the sliding surface state $\dot{S} \rightarrow 0$ in Fig. 2e as expected. Conclusively, the designed observer works well as a secular disturbance detector.

\subsection{Oscillating Disturbance}

As satellite rotates around the earth, it may be affected by oscillating disturbances like aerodynamic force and earth oblateness, etc. For the precision attitude control, the oscillating disturbance needs to detect. The performance of detecting the oscillating disturbance depends on the observer processing frequency. For the detection of high frequency disturbance, the observer frequency shall be greater 5 $\sim 10$ times than the disturbance frequency for more precise prediction. Assume the oscillating disturbance $u=\left[\begin{array}{lll}0 & 0.1 \sin 2 \pi f_{D} t & 0\end{array}\right]$ acts on the satellite during time $t=(20,60) \mathrm{sec}$ on $y$-axis. Here, the external disturbance frequency $f_{D}=5 \mathrm{~Hz}$, and the observer is then set to process every $0.02 \mathrm{sec}$, i.e., 10 times faster than the disturbance frequency. The time constant of error $e$ shall be then reduced by using bigger gain $c$. As shown in Fig. 3, the oscillating disturbance in Fig. $3 \mathrm{a}$ is well detected and the magnitude is estimated exactly as in Fig. 3b.

\section{Actuator Fault Detection and Identification}

We consider now the application of the above continuous observer on the detection and identification of a fault, e.g. momentum wheel motor speed fluctuation in a CMG.

\subsection{Disturbances Due to Momentum Wheel Speed Fault}

The required torque for a spacecraft maneuver is generated by steering the gimbal of each CMG. To generate the exact amount of required torque, $\mathrm{CMG}$ wheel momentum should be maintained constant in a nominal value. When a malfunction occurs in the wheel speed controller or bearing friction, etc., then the wheel speed might fluctuate and the disturbances are generated. The speed fluctuation can be detected by using the same disturbance detector suggested above.

Assume that the speed control of wheel No. 1 in Fig. 1 malfunctions. The wheel speed fluctuates as $\dot{h}=\left[0.01 \times 2 \pi f_{d} \cos 2 \pi f_{d} t \quad 0 \quad 0 \quad 0\right]^{T} \quad$ where $f_{\mathrm{d}}=1 \mathrm{~Hz}$ during the maneuver time $t=(20,40) \mathrm{sec}$ interval. Even though the wheel speed fluctuates and thus induces the oscillating disturbance torque on the spacecraft body as $u_{f}=-A \dot{h}$, the spacecraft attitude 


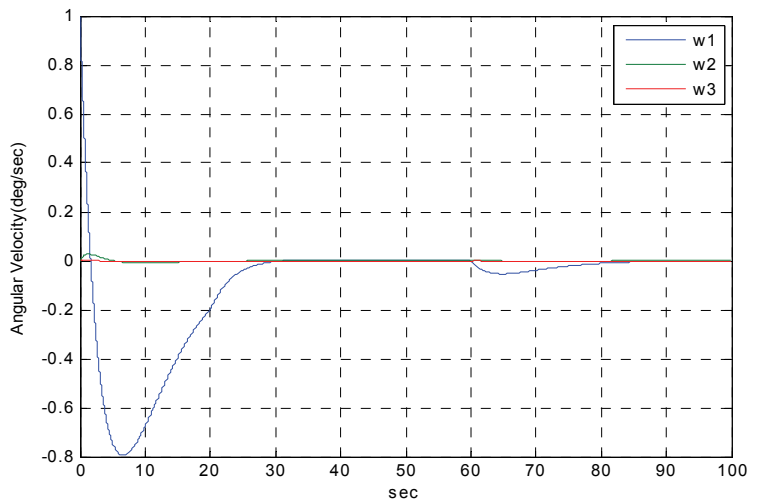

(a)
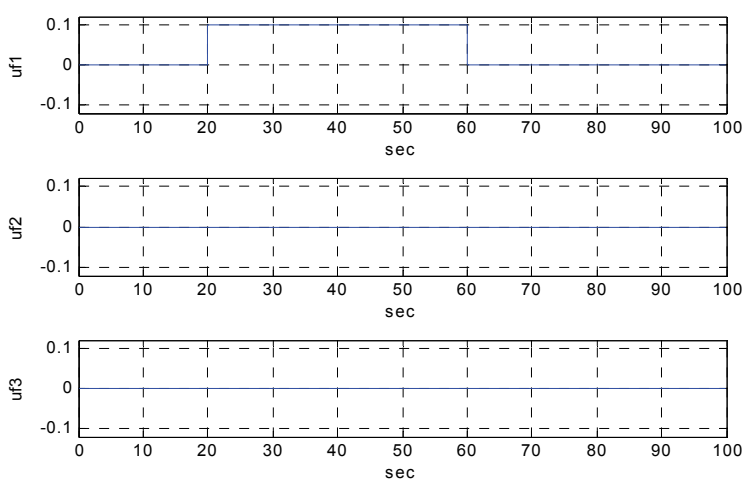

(c)
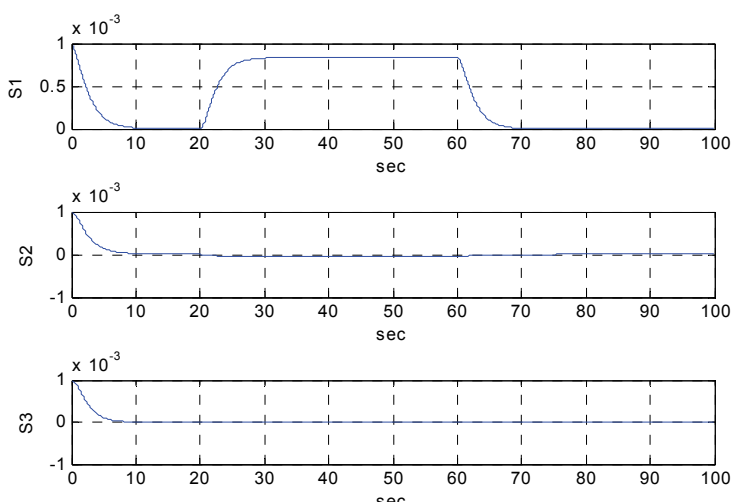

(e)

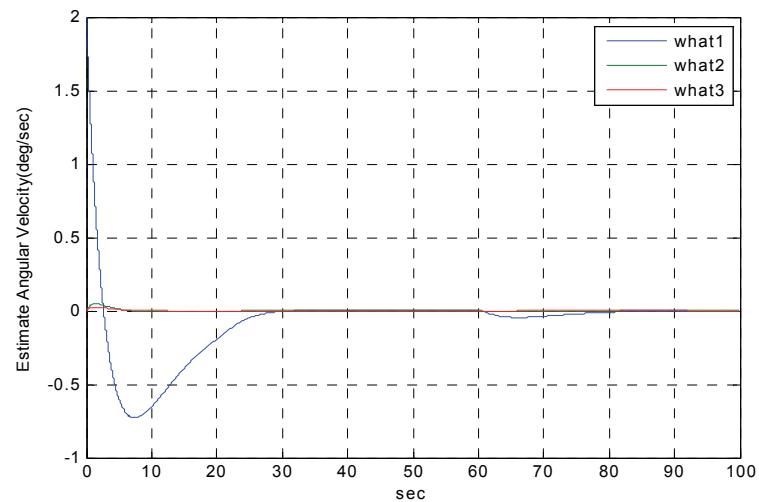

(b)
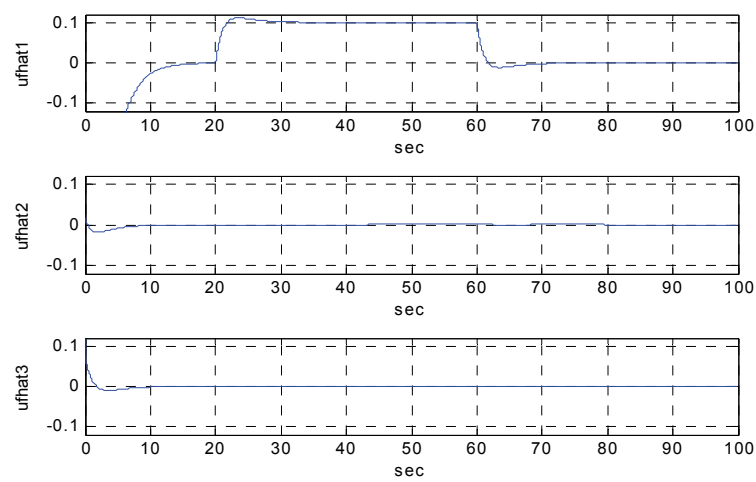

(d)

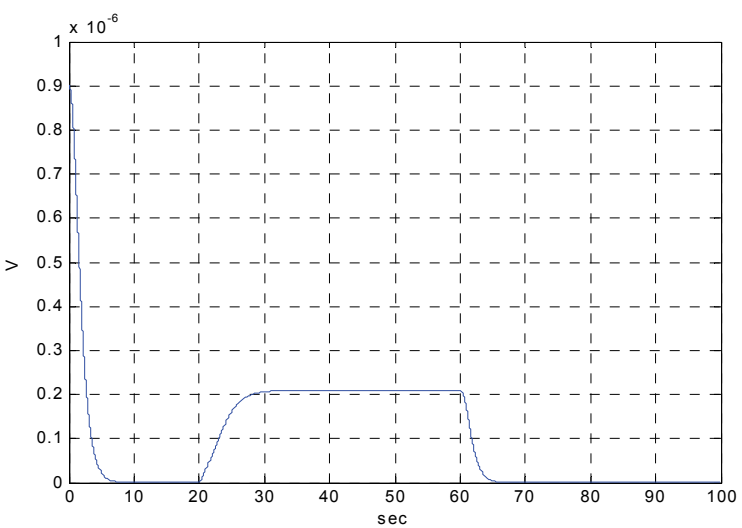

(f)

Fig. 2 Responses on a secular disturbance. 

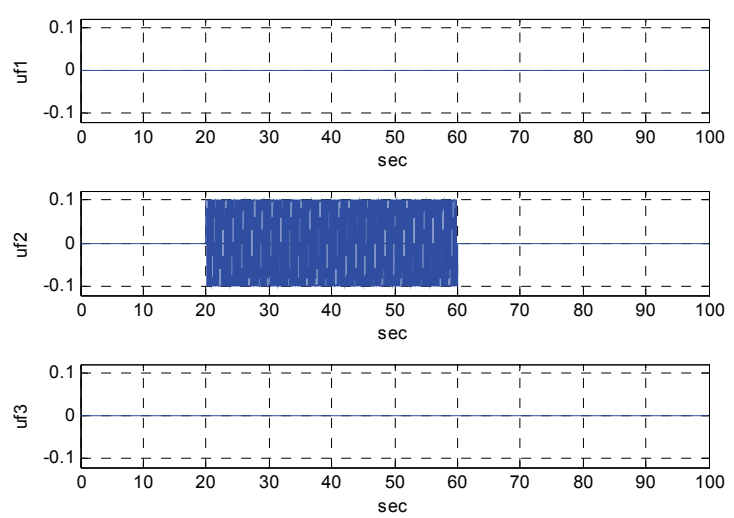

(a)

Fig. 3 Responses on oscillating disturbance.

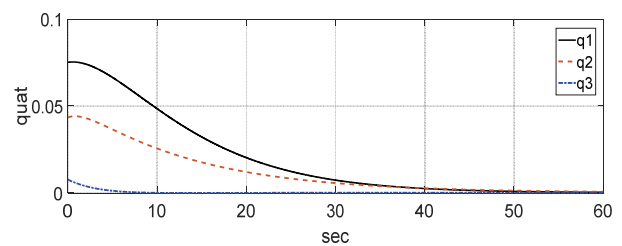

(a)

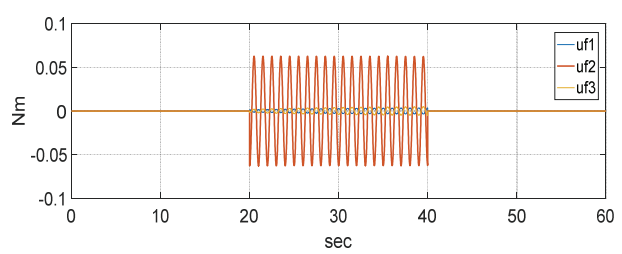

(c)

Fig. 4 Observer responses to a momentum wheel speed fault.

is finally stabilized as shown in Fig. 4a. The angular velocity of the spacecraft is stabilized to the origin as shown in Fig. 4b. The angular velocity estimate $\widehat{\omega}$ starts from an arbitrarily initial guess but aptly follows the real angular velocity $\omega$, as before. The disturbance inserted from $t=20 \mathrm{sec}$ in Fig. $4 \mathrm{c}$ is detected immediately with the magnitude identified exactly as in Fig. 4d. The designed observer works well as a oscillating disturbance detector.

\subsection{Fault Identification Algorithm of Malfunction Wheel}

The wheel induced disturbance torque is propagated to the all three body axes. The disturbances are thus detected in all three axes by the disturbance observer, as shown in Fig. 4d. This is due to the change of CMG
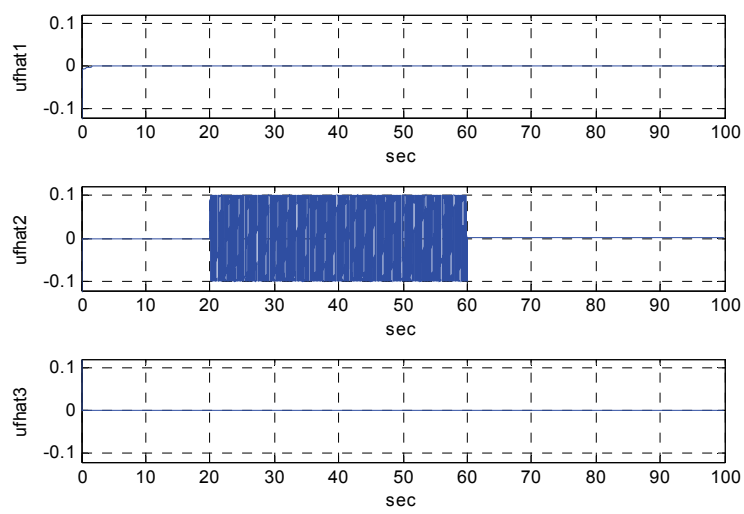

(b)

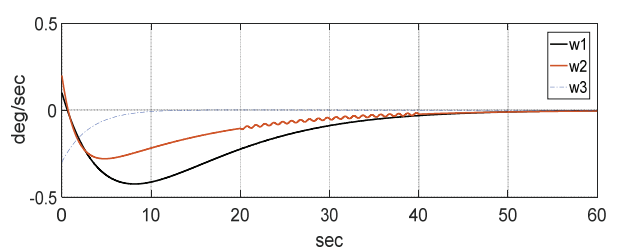

(b)

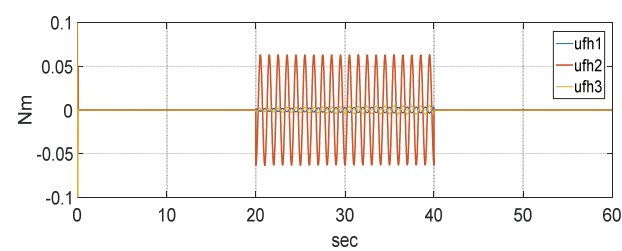

(d)

gimbal angles during maneuvers. The spacecraft operator needs to identify the abnormal wheel among the normal wheels. The disturbance effects on the spacecraft body depend on the instantaneous gimbal orientation, written as $u_{f}=-A \dot{h}$. The disturbance estimate $\hat{u}_{f}$ is obtained by the disturbance detector as before. To identify the malfunction wheel, $\widehat{u}_{f}$ is projected on the column space of $D$ and $A$ as

$$
\begin{aligned}
& \dot{\hat{h}}=A^{T} \hat{u}_{f} \\
& \dot{\hat{h}}_{N}=D^{T} \hat{u}_{f}
\end{aligned}
$$

We can obtain the candidate responses for $\hat{\dot{h}}$, as shown in Fig. 5a. However, ambiguity still exists as to which wheel-No. 1 or No. 3 in this simulation-is malfunctioning. From the fact that $\hat{u}_{f}$ is maximally projected on the wheel axis as in Eq. (11) and 

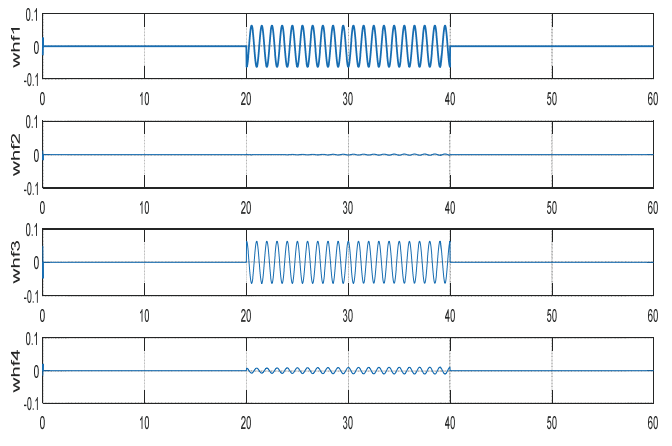

(a)

Fig. 5 Fault wheel identification.

minimally projected (i.e., orthogonal) to the output torque axis as in Eq. (12) of the malfunctioning wheel, by choosing the wheel axis with the direction nearest to $\hat{u}_{f}$, we can finally isolate No. 1 as the fault wheel, as shown in Fig. 5 b.

\section{Hardware Simulator Verification}

We now verify the performance of the fault detector with the hardware ground simulator, i.e. an air-bearing test platform recently designed and manufactured at Spacecraft Control Laboratory in Korea Aerospace University as Fig. 6. The simulator has a table-top configuration capable of floating $100 \mathrm{~kg}$-class platform, the scale model for a mid-class satellite. System MOI is designed as $I=\operatorname{diag}\{20,15,15\}, 1 / 10$ scale model of mid-class spacecraft. Attitude is measured by reference sensors: a sun-sensor and an accelerometer, which simulate the sun vector and earth vector, respectively. The angular velocity is measured by gyros. All sensors and actuators are not for the space flight class, but for the ground vehicle class with lower grade. CMGs are developed in SCL KAU as Fig. 7. Each CMG has a momentum wheel of 2/3 Nms angular momentum and a gimbal with maximum acceleration of $3 \mathrm{rad} / \mathrm{sec}$.

\subsection{Fault Identification of Malfunction Momentum Wheel}

The fault is so injected on the momentum wheel

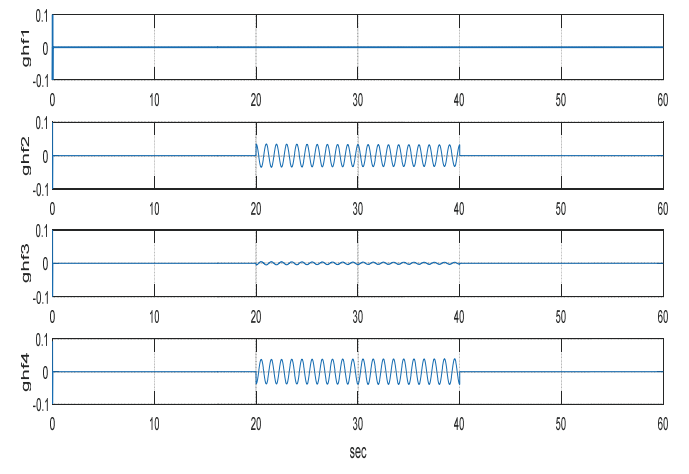

(b)

speed control of CMG1 that the wheel speed fluctuates as $\dot{h}=\left[h_{o} \times 2 \pi f_{d} \cos 2 \pi f_{d} t \quad 0 \quad 0 \quad 0\right]^{T}$ where $f_{\mathrm{d}}=0.1$ $\mathrm{Hz}$ at time $t=10 \mathrm{sec}$ as in Fig. 8. The disturbance detection algorithm detects the disturbance as in Fig. 9. We can see that all body axes are fluctuated by disturbance torque. Thereafter, the fault identification algorithms of Eqs. (11) and (12) are applied to identify the fault wheel among four wheels. By projection of the

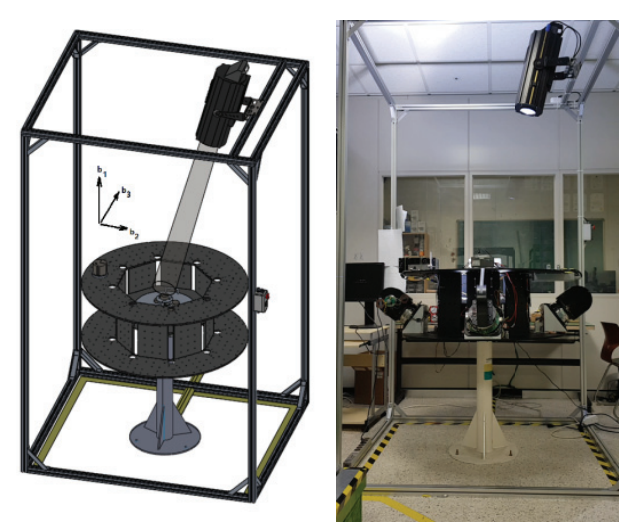

Fig. 6 Hardware simulator on an air-bearing.

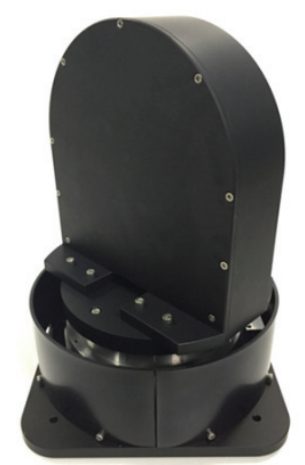

Fig. 7 SCL KAU CMG. 


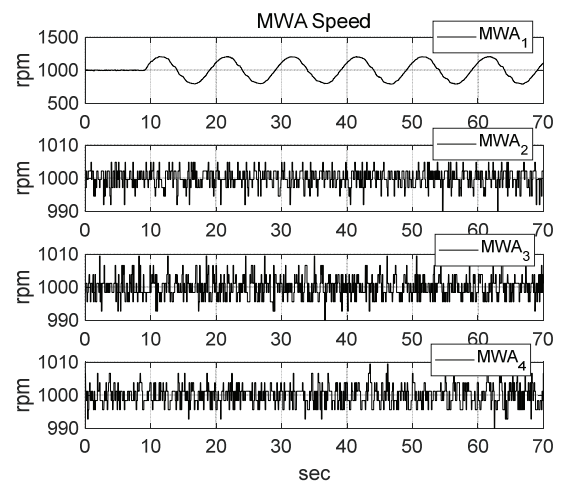

Fig. 8 MWA speed.

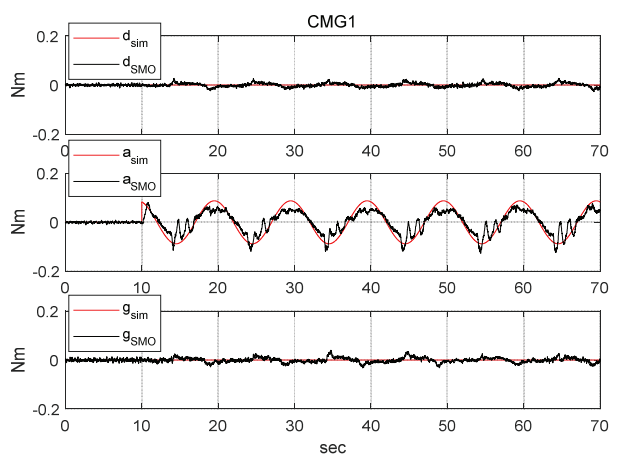

(a)
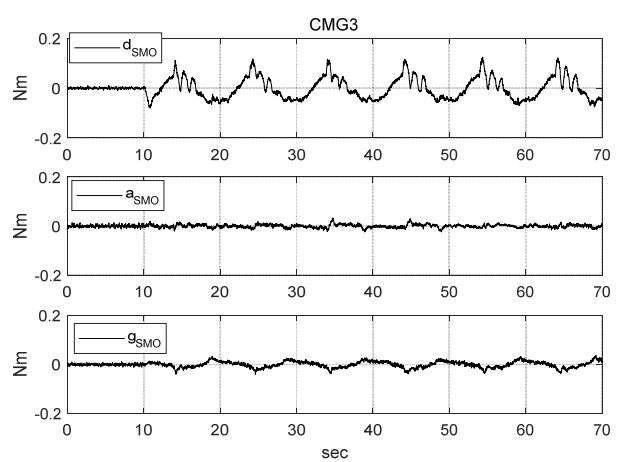

(c)

Fig. 10 Fault wheel identification.

estimated disturbance on each CMG frame, the comparison of four results in Fig. 10 leads to the final conclusion that there exists the speed fault in momentum wheel of CMG1.

\section{Conclusion}

Application of a continuous nonlinear second order sliding mode observer is suggested to detect disturbances in spacecraft with CMGs. Both slowly and highly oscillating external disturbances are aptly
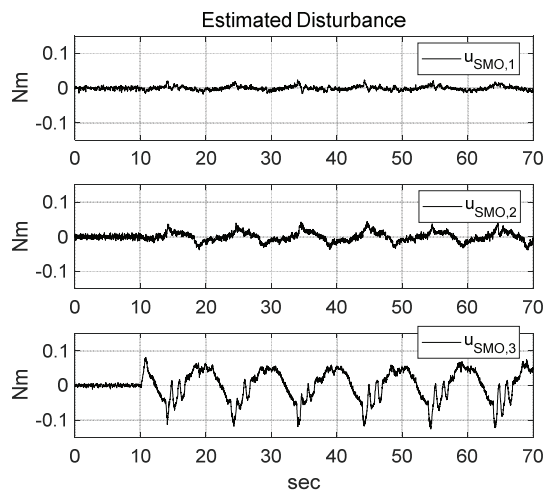

Fig. 9 Estimated disturbances.

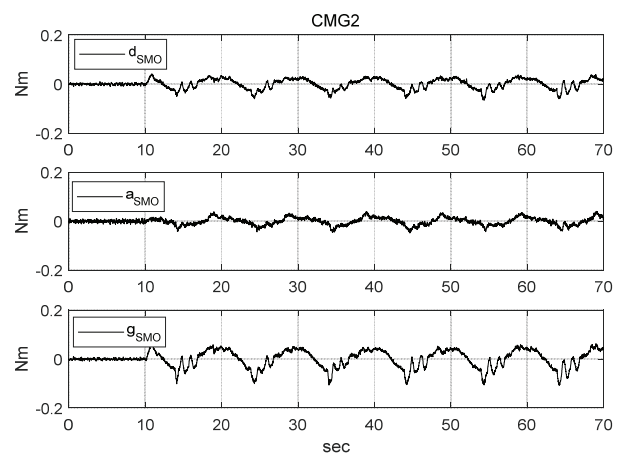

(b)
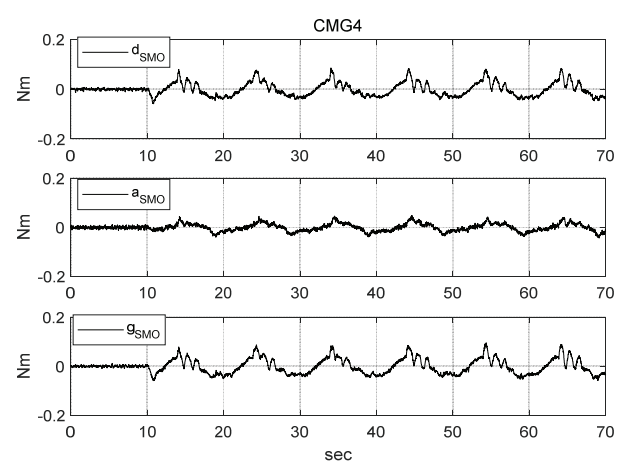

(d)

detected, and the magnitude is identified successively. Internal wheel faults that result from wheel speed error can be detected using the suggested observer and the fault $\mathrm{CMG}$ can be identified by applying the simple vector transpose identifying logic. The algorithm performance is verified in real hardware simulator.

\section{Acknowledgements}

This work was supported by the GSRC (Global 
Surveillance Research Center) program funded by the DAPA (Defense Acquisition Program Administration) and ADD (Agency for Defense Development).

\section{References}

[1] Sidi, M. 1997. Spacecraft Dynamics and Control. Cambridge University Press.

[2] Masterson, R., et al. 2002. "Development and Validation of Reaction Wheel Disturbance Models: Empirical Model." Journal of Sound and Vibration 249 (3): 575-98.

[3] Taniwaki, S., and Ohkami, Y. 2003. "Experimental and Numerical Analysis of Reaction Wheel Disturbance." JSME International Journal 46 (2): 519-26.

[4] Oh, H., et al. 2001. "Torque and Force Measurement of a Prototype KAU Reaction Wheel and the Effect of Disturbance on the Attitude Stability of Spacecraft." Journal of Mechanical Science and Technology 15 (6): 743-51.

[5] Venkatasubramanian, V., et al. 2003. "A Review of Process Fault Detection and Diagnosis Part I; Quantitative Model Based Methods." Computers and Chemical Engineering 27: 293-311.

[6] Chen, R., et al. 2006. "Health Monitoring of a Satellite System." Journal of Guidance, Control and Dynamics 29 (3): 593-605.

[7] Ahmadi, B., and Namvar, M. 2012. "Robust Detection and Isollation of Failures in Satellitte Attitude Sensors and Gyro." Robotica 30 (7): 1157-66.

[8] Chen, W., and Saif, M. 2001. "Robust Fault Detection in Uncertain Nonlinear Systems via a Second Order Slding
Mode Observer." In Proceedings of the 40th IEEE Conference on Decision and Control 1: 573-8.

[9] Jiang, T., and Khorasani, K. 2007. "A Fault Detection, Isolation, and Reconstruction Strategy for a Satellite's Attitude Control Subsystem with Redundant Wheels." IEEE International Conference on Systems: 3146-52.

[10] Slotine, J., and Li, W. 1991. Applied Nonlinear Control. Prentice Hall.

[11] Oh, H., and Kim, J. 2014. "The Detection and Identification of Control Moment Gyro Faults." IAA-AAS-DyCoSS2-14-13-05, Rome.

[12] Oh, H., and Cheon, D. 2005. "Precision Measurements of Reaction Wheel Disturbances with Frequency Compensation Process." Journal of Mechanical Science and Technology 19 (1): 136-43.

[13] Wie, B., Weiss, H., and Arapostathis, A. 1989. "Quaternion Feedback Regulator for Spacecraft Eigenaxis Rotations." Journal of Guidance, Control and Dynamics 12 (3): 375-80.

[14] Oh, H. S., and Vadali, S. 1991. "Feedback Control and Steering Laws for Spacecraft Using Single Gimbal Control Moment Gyros." Journal of Astronautical Science 39: 183-203.

[15] Oh, H. S., Lee, J. H., and Lee, H. J. 2018. "Mass Properties Estimation of a Satellite Simulator Based on the Integration Method." The 10th International Conference for Mechanical and Electrical Technology, Vancouver.

[16] Kim, J. K., Lee, J. Y., Lee, J. H., and Oh, H. S. 2016. "Disturbance Detection of Control Moment Gyros and Identification of Gimbal Fault." The 6th International Congress for Technical Diagnosis, Gliwice. 